\title{
Research Paper \\ The Relationship Between Electromagnetic Waves and Melatonin Hormone Levels in Golestan Province Power Substations
}

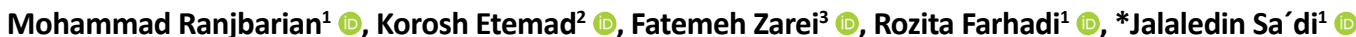

1. Department of Occupational Health and Safety Engineering, School of Public Health and Safety, Shahid Beheshti University of Medical Science, Tehran, Iran. 2. Department of Epidemiology, School of Public Health and Safety, Shahid Beheshti University of Medical Science, Tehran, Iran.

3. Department of Occupatonal Health and Safety Engineering, School of Public Health, Alborz University of Medical Science, Karaj, Iran.

\begin{tabular}{|c|c|}
\hline $\begin{array}{l}\text { Use your device to scan } \\
\text { and read the article online }\end{array}$ & Citaton: Ranjbarian M, Etemad K, Zarei F, Farhadi R, Sa'di J. [Evaluation of the Relationship Between Electromagnetic \\
\hline aptista & $\begin{array}{l}\text { Waves and Melatonin Hormone Levels in Golestan Power Substations and Comparison With the Control Group in } 2016 \text { (Per- } \\
\text { sian)]. Journal of Arak University of Medical Sciences (JAMS). 2021; 24(1):74-83. https://doi.org/10.32598/JAMS.24.1.3931.1 }\end{array}$ \\
\hline afirtis & dol'https://doi.org/10.32598/JAMS.24.1.3931.1 \\
\hline
\end{tabular}

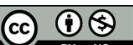

Article Info:

Received: 04 May 2020 Accepted: 29 Nov 2020 Available Online: 01 April 2021

Keywords: Radiation, Electromagnetic radiation, Melatonin

\section{A B STRACT}

Background and Aim It is almost impossible to imagine life without electromagnetic waves. Such waves play a key role in the function of melatonin and create numerous diseases.

Methods \& Materials This descriptive-analytical study was conducted on 230 kV substations in Golestan Province, Iran in 2016. In total, 44 workers of electrical substations (the case group) and 23 workers of healthcare centers (the control group) with 24-hour shift works were enrolled in this study.

Ethical Considerations This study was approved by the Ethics Committee of Shahid Beheshti University Medical Sciences (Code: IR.SBMU.THNS.REC.1395.9).

Results The obtained data suggested that $100 \%$ of the electric field intensity and the magnetic flux density measured at the substations were within the permissible range of occupational exposure. The mean level of melatonin in the case group was lower than that in the control group; however, no significant difference was observed in this regard $(P=0.761)$. Additionally, there was no significant difference between melatonin level and age $(P=0.381)$, work experience $(P=0.213)$, job groups $(P=0.515)$, and employee activity area $(\mathrm{P}=0.482)$.

Conclusion According to this study, no significant relationship was observed between electromagnetic waves and melatonin levels. Moreover, electromagnetic waves were within the permissible exposure. Thus, electromagnetic waves did not affect the level of melatonin hormone in male employees of 230 kV substations in Golestan Province. However, there was a decrease in the case group, compared to the controls. Therefore, definite comments require further investigation.

\section{Extended Abstract}

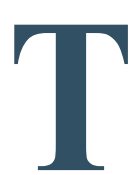

\section{Introduction}

he effects of electromagnetic fields on living organisms at different frequencies have long been studied by researchers and in numerous cases. Moreover, the long duration of studies and the slow impact of fields on life cycle processes prevent making a definite statement. Accordingly, the judgment was based only on laboratory findings on animal samples. However, there exists a consensus on the adverse effects of too much exposure to a magnetic field. Necessary information about the level of melatonin in the employees of power sub-

\section{*Corresponding Author:}

Jalaledin Sa'di, MSc.

Address: Department of Occupational Health and Safety Engineering, School of Public Health and Safety, Shahid Beheshti University of Medical Science, Tehran, Iran. Tel: +98 (21) 22432040

E-mail: jalalsaadi@yahoo.com 
stations in Iran is scarce. Besides, exposure to electromagnetic waves is harmful. Thus, this study aimed to determine the relationship between electromagnetic waves and serum melatonin levels in the employees of $230 \mathrm{kV}$ substations in Golestan Province, Iran.

\section{Materials and Methods}

This descriptive study was performed on male employees of $230 \mathrm{kV}$ substations in Golestan Province, Iran, in 2016. The intensity of electric and magnetic fields in five 230 $\mathrm{kV}$ substations in Golestan Province was determined and the relevant serum melatonin levels were measured. Demographic information was collected by a questionnaire. Changes in melatonin hormone levels of 44 employees were studied as the target group and compared with the control group (23 guards working in healthcare centers).

The obtained data were analyzed in Spss v. 22. Quantitative variables were reported as mean and standard deviation and qualitative variables as a percentage. The KolmogorovSmirnov test was used to establish the normality of the data at a significance level of 0.05 . Based on the collected results, parametric and non-parametric tests were selected. Independent Samples t-test, Mann-Whitney U test, one-way Analysis of Variance (ANOVA), as well as Kendall and Pearson correlation coefficient tests were used to compare melatonin levels in the case (operator, substation guards) and control (healthcare center staff) groups.

\section{Results}

The mean electric field strength measured at substations was equal to $5.91 \mathrm{~V} / \mathrm{m}$ in the range of 7.55-5.44; the average magnetic flux density was $5.08 \mathrm{mG}$ in the range of 6.54-0.25. These values fell in the permissible range of occupational exposure. The Mean \pm SD level of melatonin hormone was calculated to be $25.4460 \pm 1.60$ and $24.58 \pm 2.45$ in the case and control groups, respectively. Independent Samples t-test data revealed no significant difference in the mean level of melatonin between the case and control groups $(\mathrm{P}=0.761)$. One-Way ANOVA data indicated no significant difference between the age groups of subjects in mean melatonin levels $(\mathrm{P}=0.381)$. In the control group, there was no significant difference between the mean melatonin levels $(\mathrm{P}=0.551)$ and age groups. Kendall correlation coefficient data suggested no significant relationship in melatonin levels and age groups between the case and control groups $(\mathrm{P}<0.05)$. One-Way ANOVA data presented no significant difference between the research groups in the mean level of melatonin $(\mathrm{P}=0.213)$. In the control group, there was no significant difference between the mean melatonin levels based on work experience $(\mathrm{P}=0.383)$. Kendall correlation coefficient results suggested no significant relationship in melatonin levels and different groups of work experience between the case and control groups $(\mathrm{P}<0.05)$. One-Way ANOVA findings signified no significant difference in the mean level of melatonin hormone in the place of activity of employees $(\mathrm{P}=0.482)$ and the occupation of individuals $(\mathrm{P}=0.515)$ between the case and control groups. Pearson correlation coefficient data revealed no significant relationship between melatonin hormone levels in the case group with the values of electric field intensity $(\mathrm{P}=0.851)$ and magnetic flux density $(\mathrm{P}=0.132)$.

\section{Discussion and Conclusion}

All explored electrical and magnetic field measurements were within national and international standards. The measurement results of this study were consistent with those of Hosseini et al. [18], Mohamadyan et al. [17], and Ghorbani et al. [20] in Iran. In their study, all measurements were within the allowable range of occupational exposure. In this study, the mean level of melatonin in the case group was slightly higher than that in the control group; no significant difference was observed between the research groups in this regard. This result was consistent with those of Juutilainen (2006) [8] and El-Hellali [9]. They also observed no significant difference in the level of melatonin in their study. However, the results of this study were inconsistent with those of Dyche [24]. The mean level of melatonin in different age groups presented no significant difference in the case-control group. A slight decrease in melatonin was observed in the control group with age; however, no decrease was observed in the case group [22]. The obtained results signified that employees who are in authorized contact with electromagnetic waves and work in a safe environment, will not be affected by these waves concerning the amount of melatonin hormone under different age conditions, exposure history, and so on. We found no association between electromagnetic waves and the hormone melatonin.

\section{Ethical Considerations}

\section{Compliance with ethical guidelines}

This study was approved by the Ethics Committee of the University of S hahid Beheshty Medical Science (Code: IR.SBMU.THNS.REC.1395.9).

\section{Funding}

The study was extracted from the MSc. thesis of the first author at the Department of Occupatonal Health and Safety Engineering, School of Public Health and Safety, Shahid Beheshti University of Medical Science, Tehran. 


\section{Authors' contributions}

Conceptualization: Mohammad Ranjbarian and Kourosh Etemad; Data analysis: Fatemeh Zarei; Research and sampling method: Jalaluddin Saadi and Rozita Farhadi; Text writing and review: All Authors.

Conflicts of interest

The authors declared no conflicts of interest. 
بررسى ارثباط امواج الكترومغناطيس با سطح هورمون ملاتونين شاغلين يستهاى برق استان

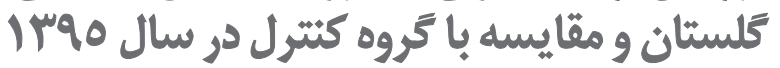

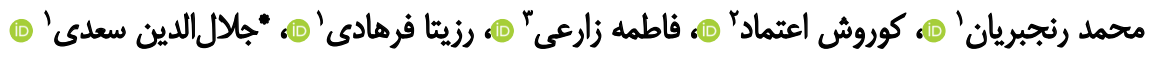

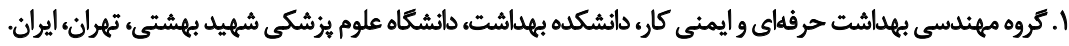

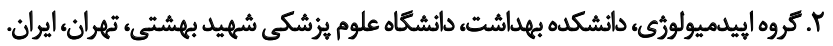

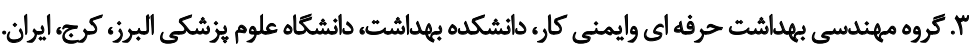

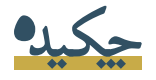

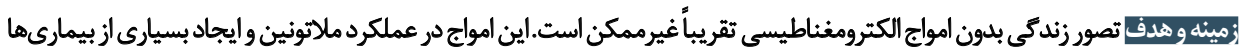

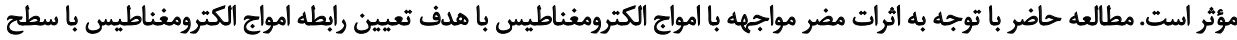

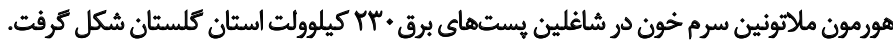

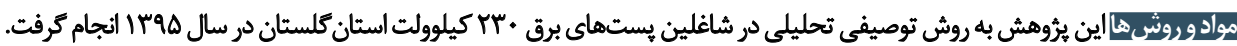

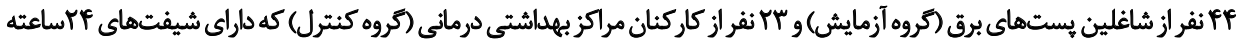

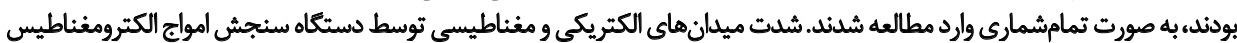

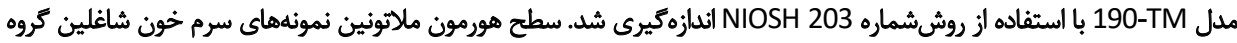

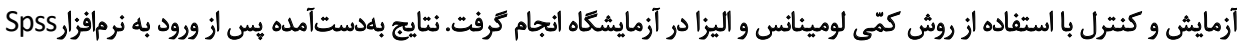

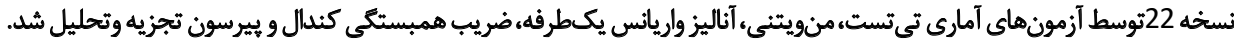

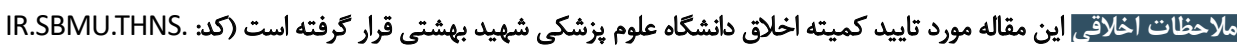

.REC.1395.9

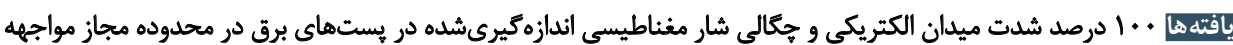

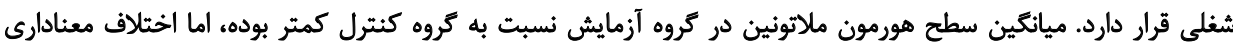

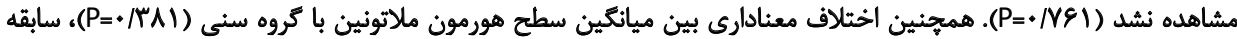

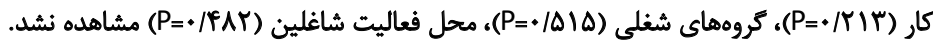

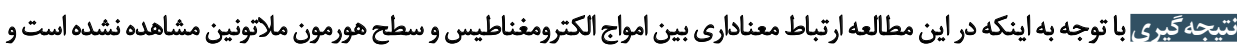

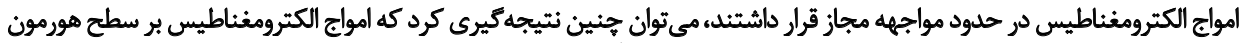

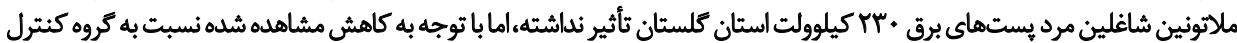
اظهار نظر قطعى نياز به بررسى هائ بيشترى برى ثاردي.
اطلاعات مقاله:

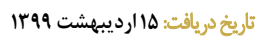

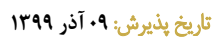

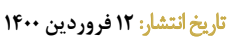

كليدوازوها: امواج الكترومغناطيسء ملاتونين

اكنون شواهدى وجود دارد مبنى بر اينكه ملاتونين در تنظيم

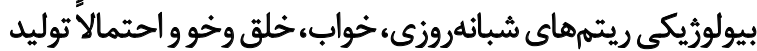

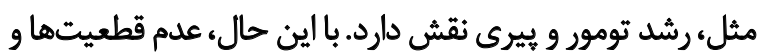

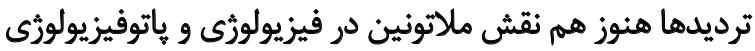

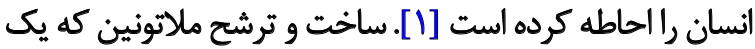

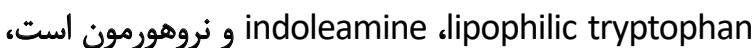

detos

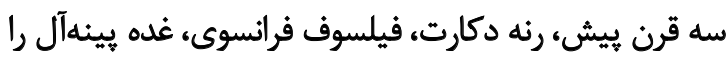

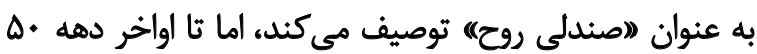

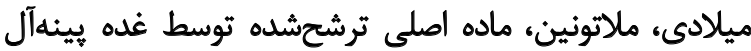
كشف نشله بود. 
قرار كرفتن در معرض ميدانهاى الكترومغناطيسى در برخى

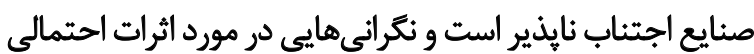

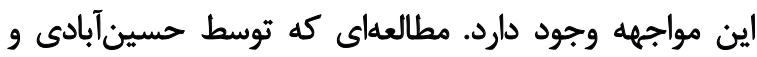

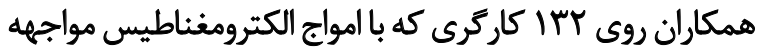

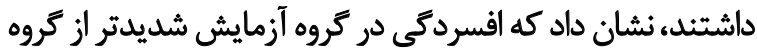

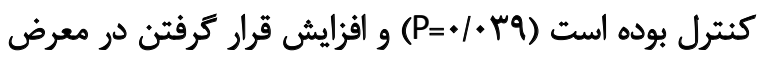

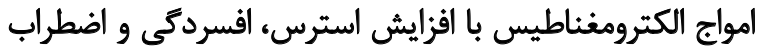

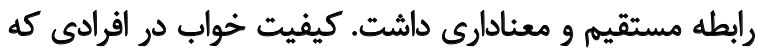

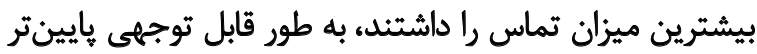

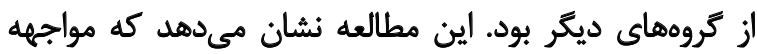

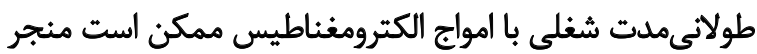
به افسردكى، استرس، اضطراب و كيفيت يايين خواب شود [11]. افراد شاغل در يستهاى برق فشار قوى نيز به نوعى تحت

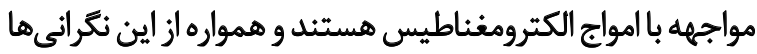

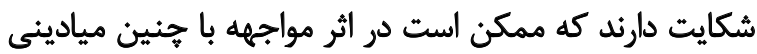

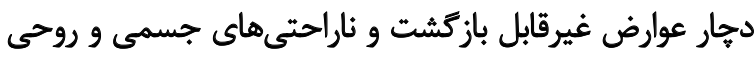

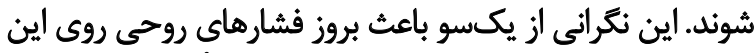

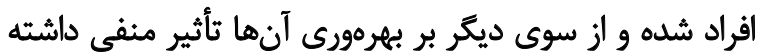

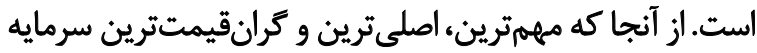

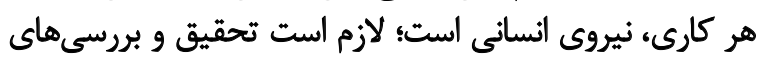

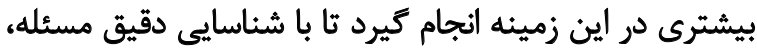

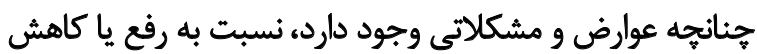

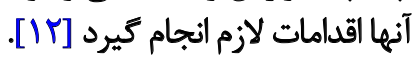

با توجه به عدم وجود اطلاعات لازم در مورد ميزان سطح

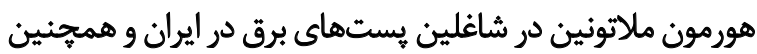

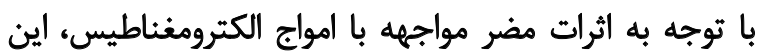

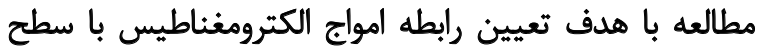

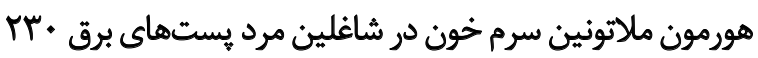
كيلوولت استان كلستان شكل ترفت

\section{مواد وروشي}

اين مطالعه توصيفى تحليلى روى fff نفر از شاغلين يستهاى

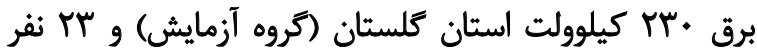

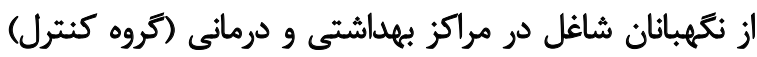

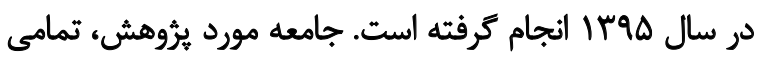

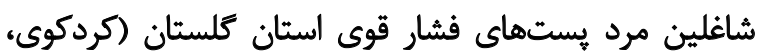

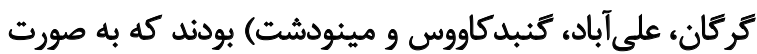
تمام شمارى وارد مطالعه شدند.

علت انتخاب شاغلين مراكز بهداشتى درمانى به عنوان كروه

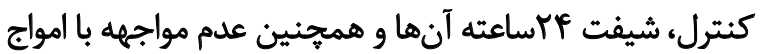

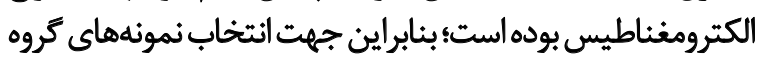

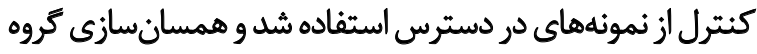
آزمايش وكنترل باتوجه به عدم همكارى و همجينين يكسانسازى إنى
توسط ثابش امواج الكترومغناطيسى در محدوده فركائس نور

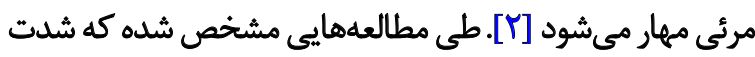

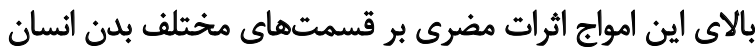

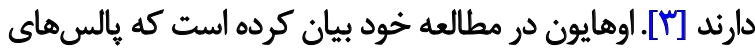

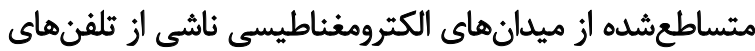

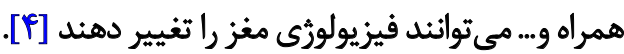

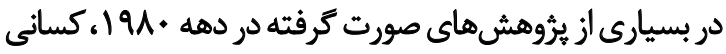

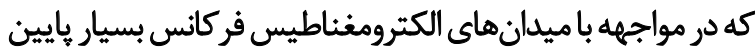
قرار داشتند، بررسى شده و و ونانسين (Extremely Low-Frequency)

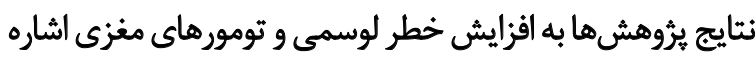

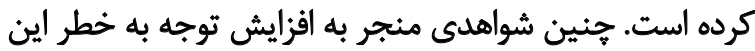

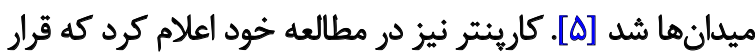

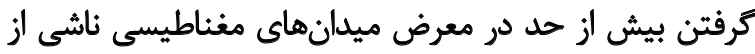

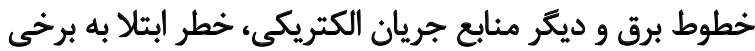

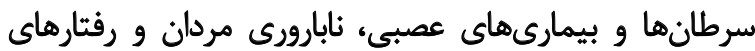
عصبى را افزايش مي دهد [عارئ.

صحرايى و همكاران در مطالعه حيوانى خود عنوان كردند كه

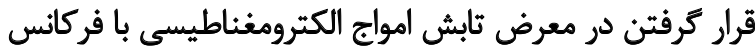

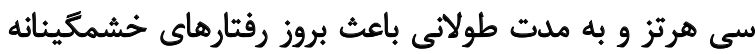

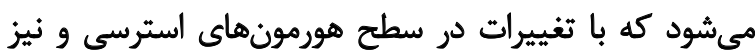
ملاتونين همراه است.

دراين آزمايش حافظه بينايى ميمون ها تخريب شده بود. ميزان

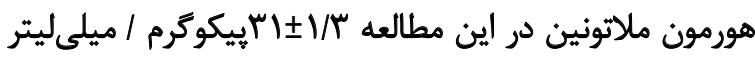

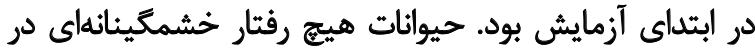

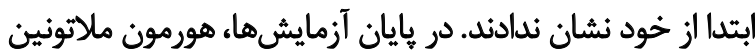

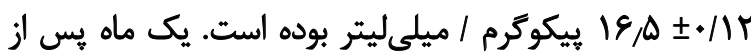

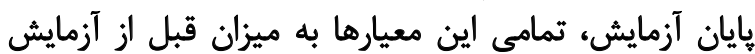

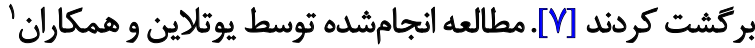

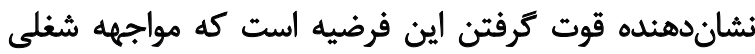

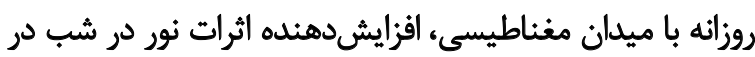

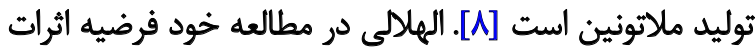

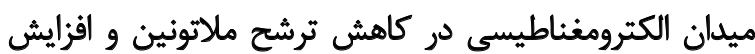

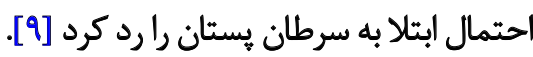

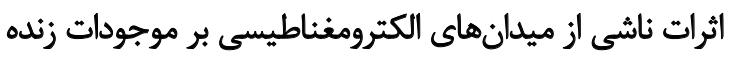

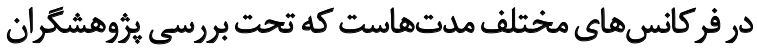

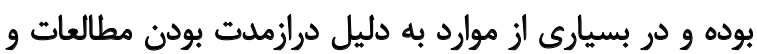

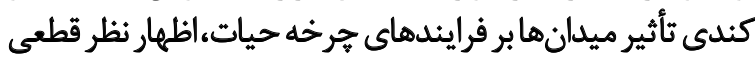

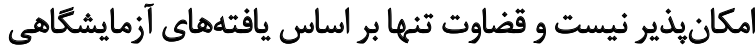

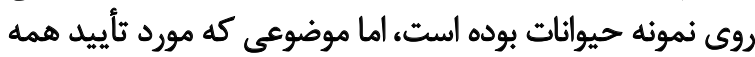

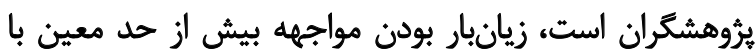

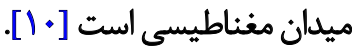

1. Juutilainen 
با سرنك و از دست افراد توسط كارشناس علوم آزمايشكاهي و

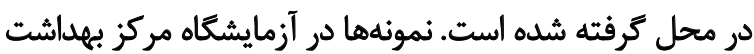

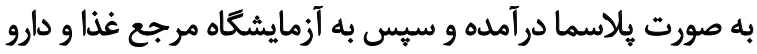

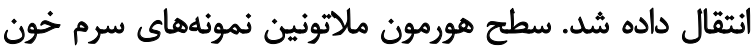

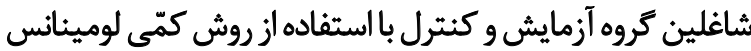

و اليزا در آزمايشكاه معتمد انجام كرفت [19 [19].

با توجه به عدم اطلاعات در مورد ميزان سطح هورمون

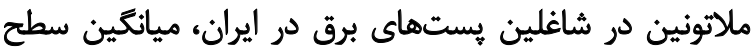

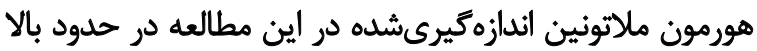

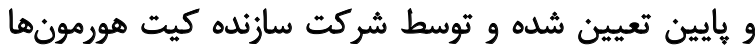

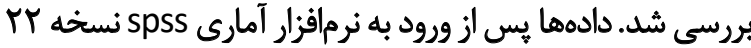

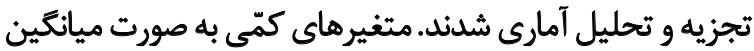

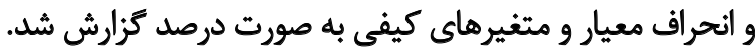

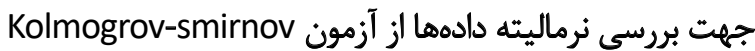

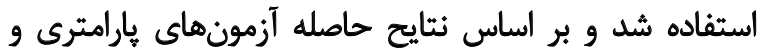
نإيارامترى متناسب انتخاب شد.

جهت مقايسه سطح هورمون ملاتونين در گروه آزمايش و

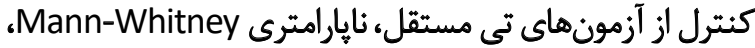

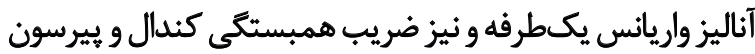

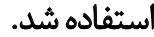

يافتهها

كروه آزمايش (ff نفر) به ترتيب در محلهاي كردكوى،

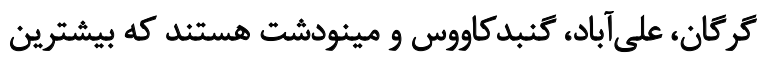

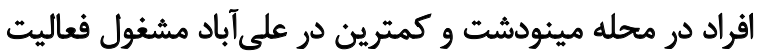

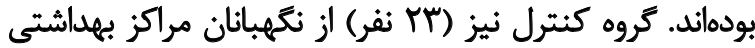

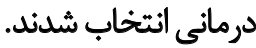

اطلاعاتجمعيتشناختى افراد(سنوسابقه كار)در جدول شماره

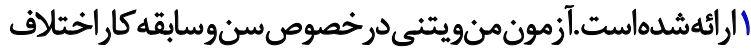

معنادارى بين كروه آزمايش و كنترل نشان نداد (ه (P> (P). مقادير تماس شغلى مجاز مواجهه با ميدانهاي مغناطيسى

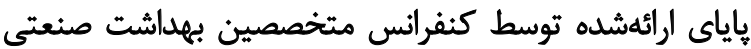

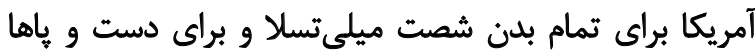

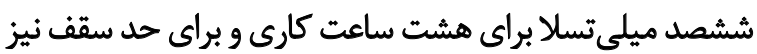

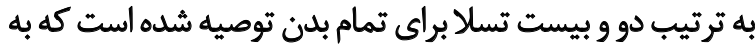
عنوان حد مجاز شغلى كشور يذيرفته شده است.

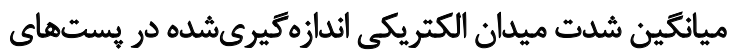

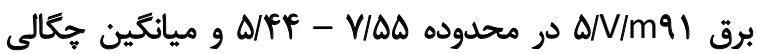

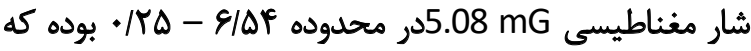

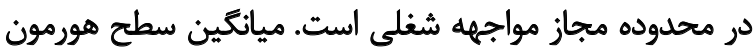

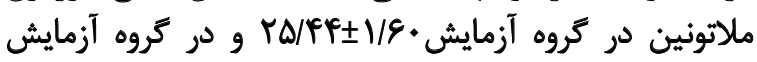

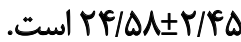

شرايط ورود به مطالعه به سختى امكانيذير بود. شرط ورود

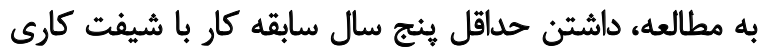

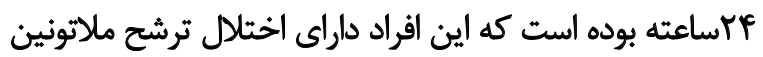

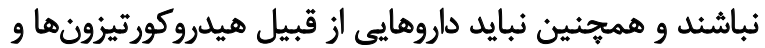

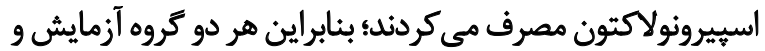

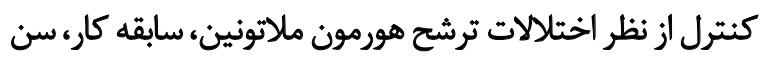

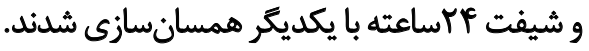

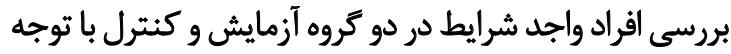

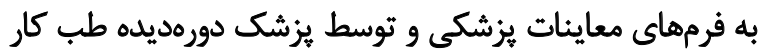

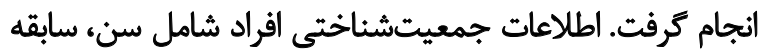

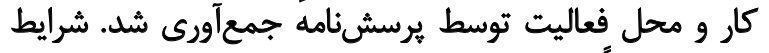

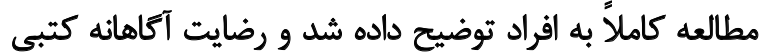

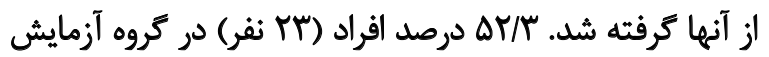

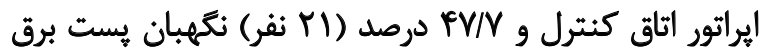

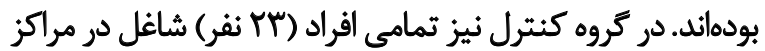

$$
\text { بهداشتى درمانى هستند. }
$$

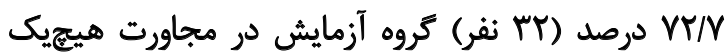

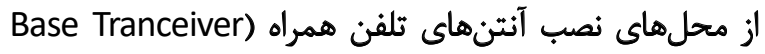

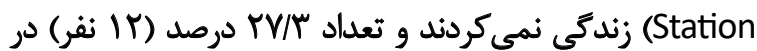

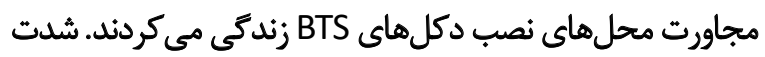

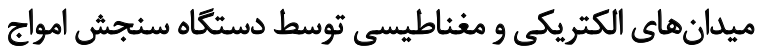

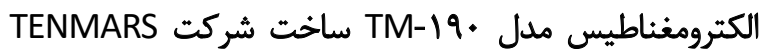

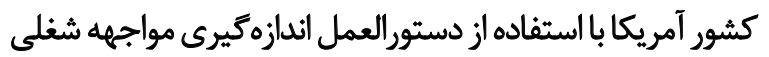

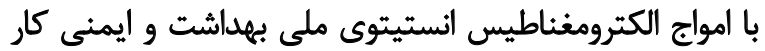

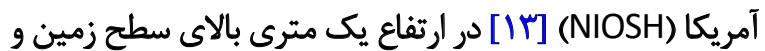

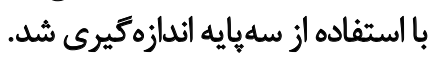

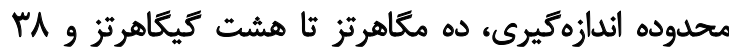

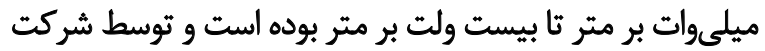

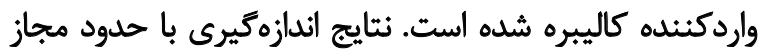

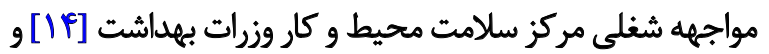

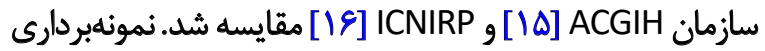
در فصل تابستان و در شرايط دمايى يكسان و در وائايان شيفت

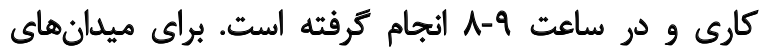

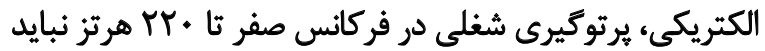

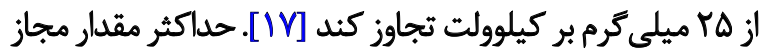

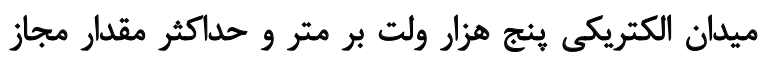

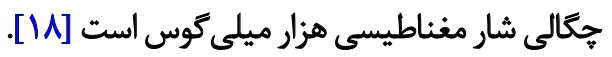

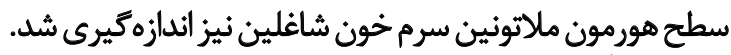

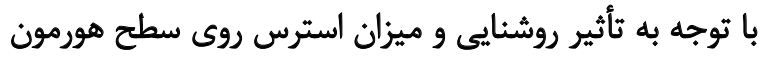

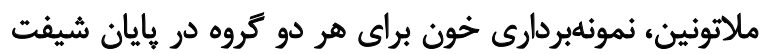

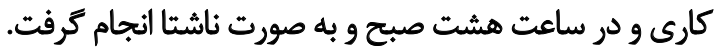
مقدار خون كرفتهشده حدود سه تا ينج سىسى بوده است كه 
جدول ا. مقايسه سن و سابقه كار كروه آزمايش و كنترل استان كلستان در سال هوبا

\begin{tabular}{|c|c|c|c|c|}
\hline \multirow{2}{*}{$\mathbf{P}$} & \multicolumn{2}{|c|}{ تعداد (درصد) } & \multirow{2}{*}{ متغير } & \\
\hline & كتثرل & آزمايش & & \\
\hline \multirow{6}{*}{. } & $8(K \& /)$ & $\varepsilon(1 H / 9)$ & $<p q$ & \multirow{6}{*}{ سن } \\
\hline & $\Delta(T M M)$ & $M(\Delta \cdot)$ & $r \cdot-m e$ & \\
\hline & $r(\mid r / 1)$ & $M(T / M)$ & TQ-MQ & \\
\hline & $V(r+/ r)$ & $(T / \pi)$ & $f=-p f$ & \\
\hline & $\cdot(\cdot)$ & $\mid(T / \pi)$ & $P Q-P q$ & \\
\hline & $r(N V)$ & $r(P / Y)$ & $>\Delta$. & \\
\hline \multirow{5}{*}{$.1 \cdot V$} & $\|(F V / A)$ & $r+(E N))$ & $\Delta-q$ & \multirow{5}{*}{ سابقه كار } \\
\hline & $\Lambda(M / A)$ & $\|(T \Delta)$ & $1 .-1 f$ & \\
\hline & $r(\mathcal{N V})$ & $\mid(T / T)$ & $10-19$ & \\
\hline & $r(N V)$ & $\cdot(\cdot)$ & $r \cdot-r F^{2}$ & \\
\hline & $\cdot(\cdot)$ & $r(r / \varepsilon)$ & $>$ PQ & \\
\hline
\end{tabular}

سابقه كار بالاترى دارند، ميانكين سطح ملاتونين خون بالاترى

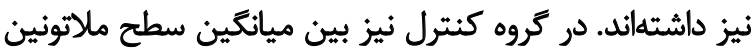

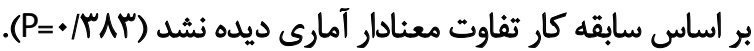

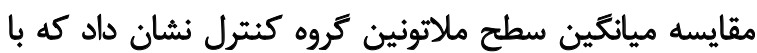

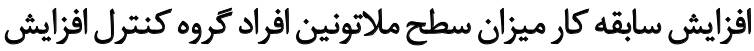
نيافته است (تصوير شماره ץ).

ضريب همبستگى كندال نيز ارتباط معنادارى بين سطح

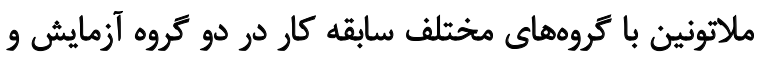

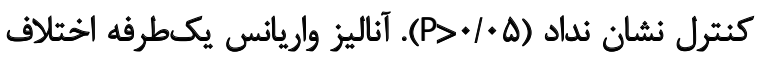
معنادارى در ميانكين سطح هورمون ملاتونين بين محل فعاليت

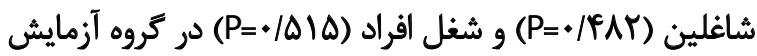

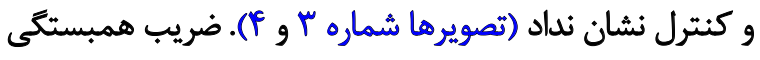

آزمون تى مستقل تفاوت معنادارى را در ميانكين سطح هورمون ملاتونين در دو كروه آزمايش و كنترل نشات نشان نداد

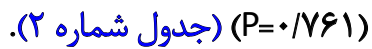

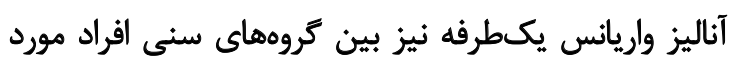

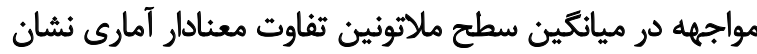

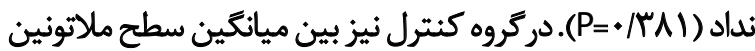

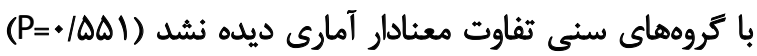

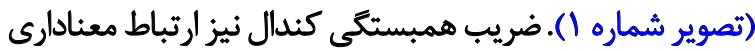

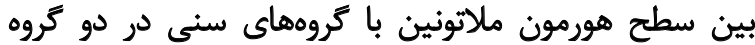

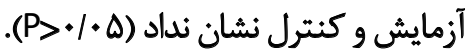

آناليز واريانس يكطرفه بين كروهانهاي سابقه كار افراد مورد

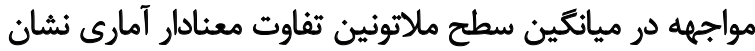
نداد (T/Y/M)

جدول r. بررسى سطح هورمون ملاتونين در كروه آزمايش و كنترل بنا به آزمون تى

\begin{tabular}{|c|c|c|c|c|c|}
\hline \multicolumn{3}{|c|}{ أزمون t } & \multirow{2}{*}{ ميانكين+|نحراف معيار } & \multirow{2}{*}{ تعداد (نفر) } & \multirow{2}{*}{ كروه - اه } \\
\hline $\mathbf{P}$ & ورجه آزادى & t معيار & & & \\
\hline \multirow{2}{*}{. NA } & \multirow{2}{*}{9} & \multirow{2}{*}{$\cdot \pi \cdot \Delta$} & $r \Delta / r F \pm V / q$. & if & آزمايش \\
\hline & & & $r F / \Delta \Lambda \pm T / F \Delta$ & זי & كتثتل \\
\hline
\end{tabular}




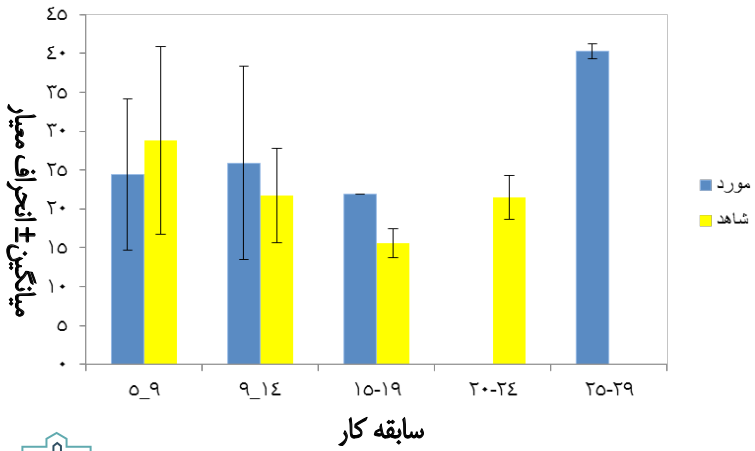

(S)

تصوير r. مقايسه سطح هورمون ملاتونين بر اساس سابقه كار در تروه آزمايش وكثترل (ميانتين بانحراف معيار)

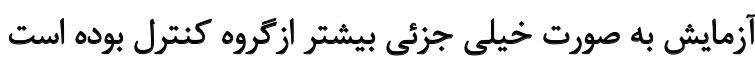

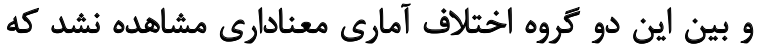

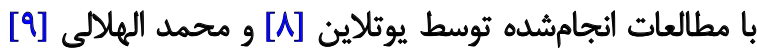

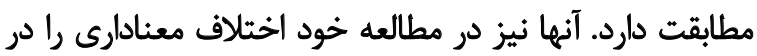
سطح هورمون ملاتونين مشاهده نكردند.

بر اساس مطالعه برينارد [بr] و نيز فيزيولوريك بدن، هورمون

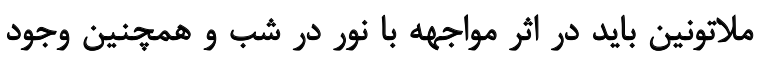

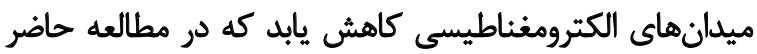

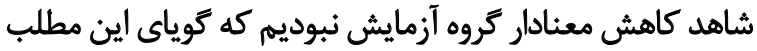

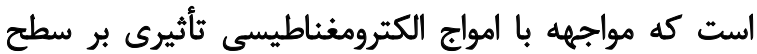

$$
\text { هورمون ملاتونين ندارد. }
$$

همجنين در اين مطالعه، ارتباطى بين مقادير ميدان الكتريكى

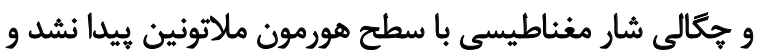

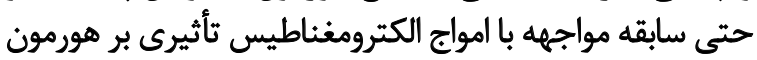
ملاتونين نداشته است كه شايد بتوان قرارئهي

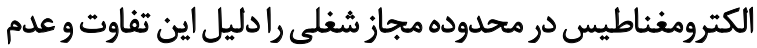

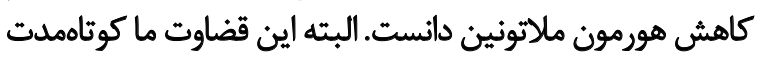

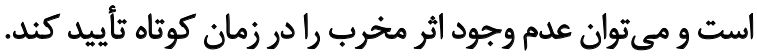
از طرفى، نتايج اين مطالعه با نتايج مطالعه ديج [آس] كه يك

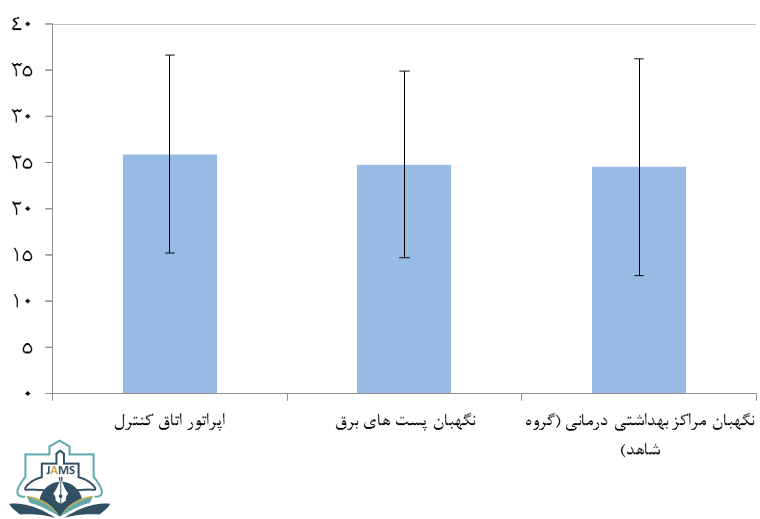

تصوير P. مقايسه سطح هورمون ملاتونين بر اساس نوع شغل در كروه آزمايش و كنترل (ميانكينثانحراف معيار)

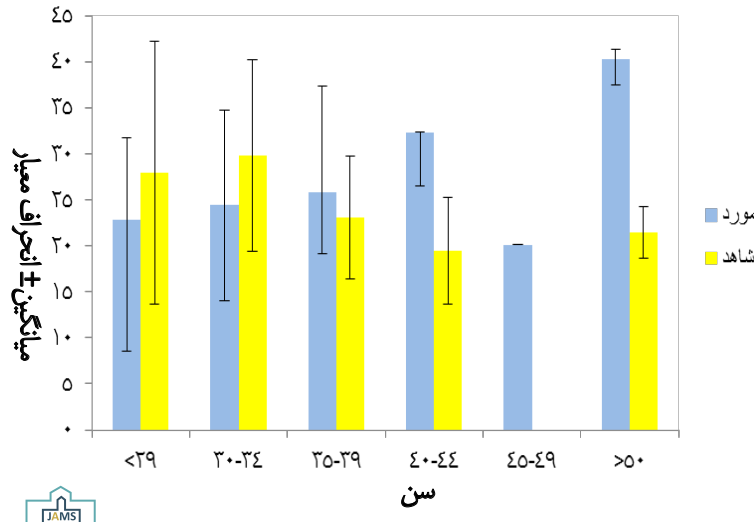

(St)

تصوير ا. مقايسه سطح هورمون ملاتوئين در ردههاي سنى مختلف در كروه آزمايش و كنترل (ميانكين

بيرسون نيز بين سطح هورمون ملاتونين در كروه آزمايش

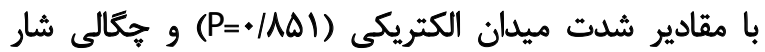
مغناطيسى (P=ائ ) ارتباط معنادارى نشان نداد. ب

تمامى اندازهكيرىهاى ميدان الكتريكى و مغناطيسى در إنائي

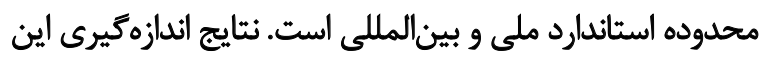

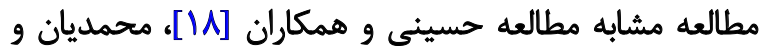
ديكران [IV] و قربانى و ديكران [•r] در ايران است. در مطالعه آنها نيز تمامى اندازمكيرىها در محدوده مجاز

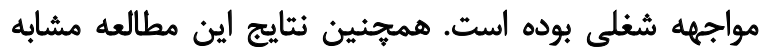

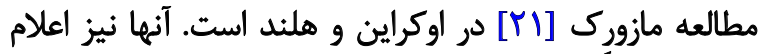

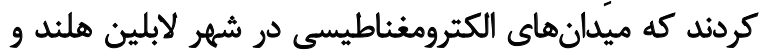
رايون اوكراين از حد مجاز تجاوز نكرده است. در اين مطالعه، ميانكين سطح هورمون ملاتونين در گروه

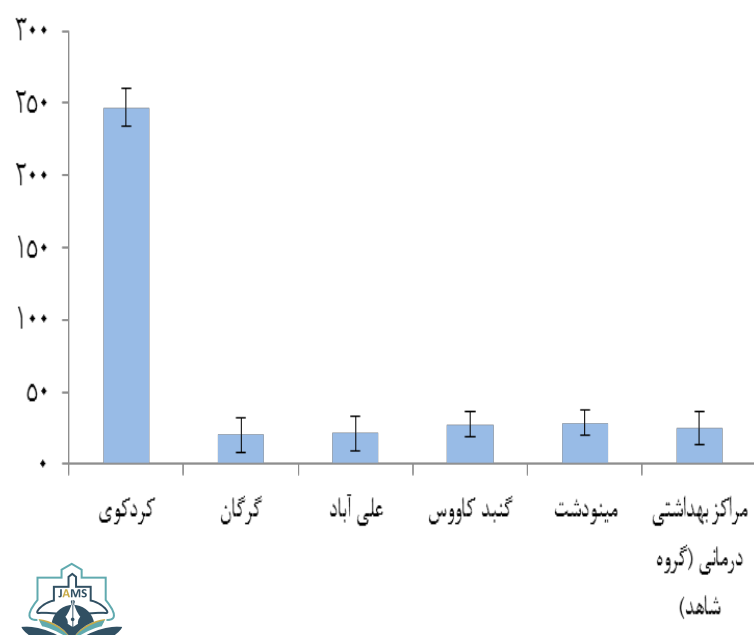

تصوير با. مقايسه سطح هورمون ملاتونين بر اساس محل هاى فعاليت در كروه آزمايش و كنترل (ميانكين بائحراف معيار) 
اضطراب عمومى در مورد عوارض بالقوه مواجهه مزمن با امواج

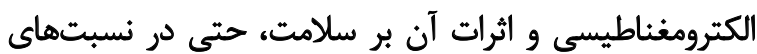
ضعيف بوده است. نتايج اين مطالعه نشان داد كه مواجهه با امواج الكترومغناطيس

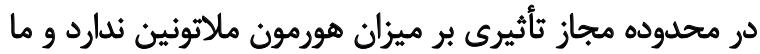

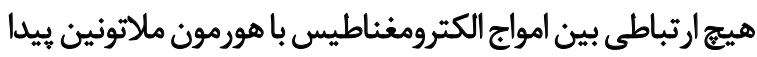

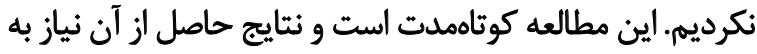

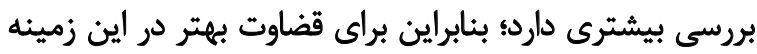

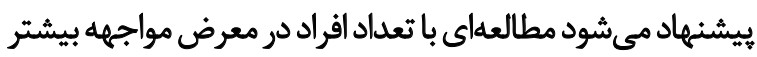
و در زمانهاى طولانىمدت (مطالعه كوهورت) صورت كيردا از طرفى، بررسى تأثير هورمون ملاتونين در شرايطى كه

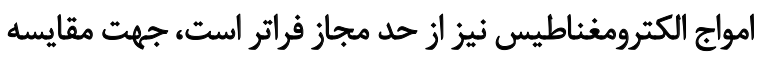

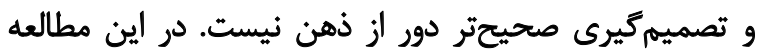

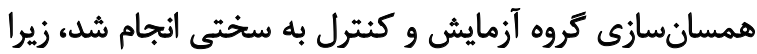

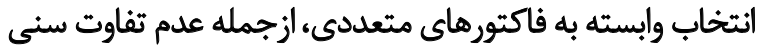

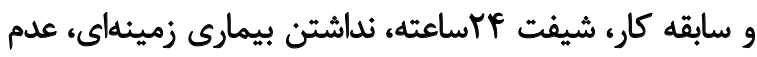

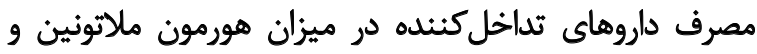

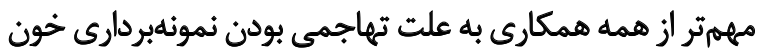

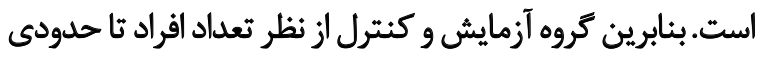
با يكديكر ثفاوت داشته، اما از نظر ساير كاير عوامل مؤثر بر بر مطالعه

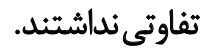

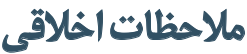

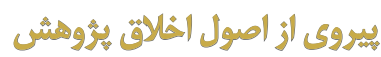

اين مطالعه توسط كميته اخلاق دانشكاه علوم بزشكى شهيد بهشتى (كد:IR.SBMU.THNS.REC.1395.9) ثأييد شد.

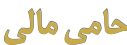

اين مطالعه از يايان نامه كارشناسى ارشد نويسنده اول در كروه

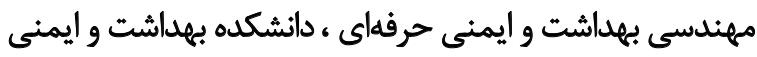
عمومى، دانشكاه علوم يزشكى شهيد بهرئ بهشتى، تهران استخراج

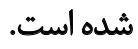

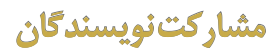

مفهوم سازى: محمد رنجبريان وكوروش اعتماد؛ تحليل دادهها:

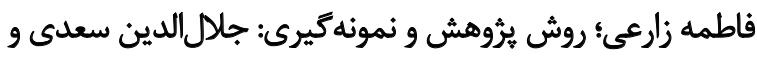
رزيتا فرهادى؛ نكارش متن و بازبينى: تمام نويسندكان.

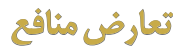

نويسئدكان مقاله هيجگونه تعارضى در منافع اعلام نكردند.
مطالعه حيوانى هست، متفاوت است. أنها در مطالعه خود بيان

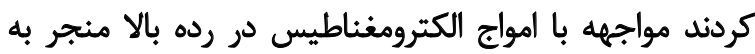

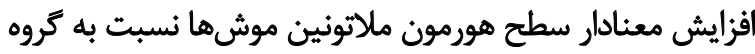

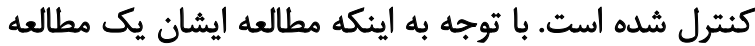

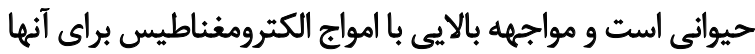

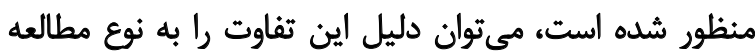

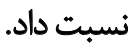

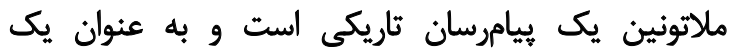

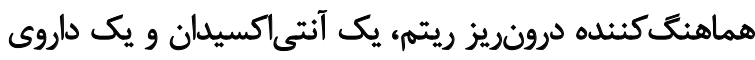

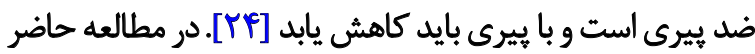

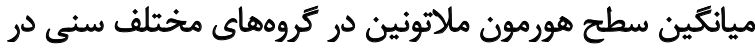
كروه آزمايش و كنترل، اختلاف معنادارى نشان نداده است.

اين مسئله به بررسى بيشترى نياز دارد. اكرجها بر اساسٍ

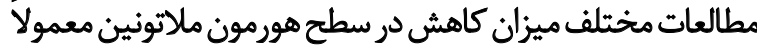

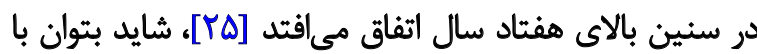

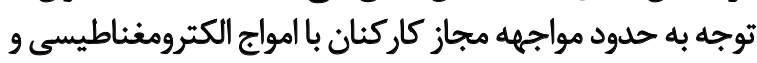
سن زير ينجاه سال اين موضوع را توجيه كرد.

نتايج اين مطالعه نشان داد كه بين سابقه كار و سطح هورمون

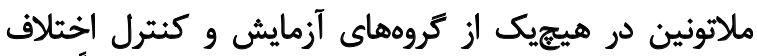

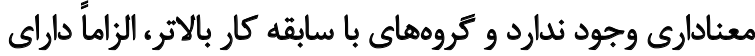
سطح هورمون ملاتونين بالاتر يا إيايينتر نيستند. متأسفانه مطالعهاي جهت مقايسه سابقه مواجهه با امواج

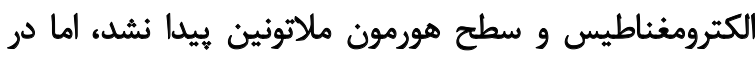

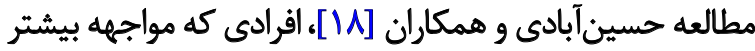

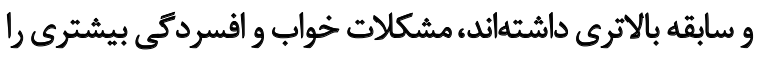

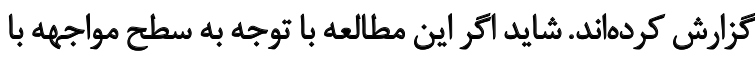

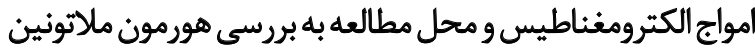

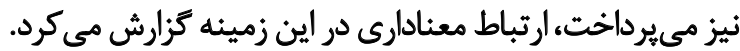

نتايج اين مطالعه ارتباط معنادارى بين شغل افراد و محل

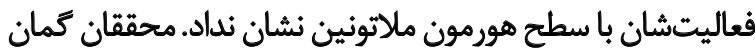

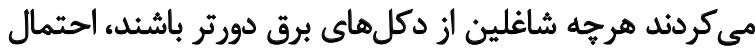

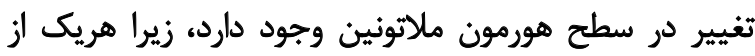

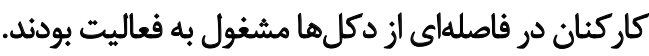

نتايج مطالعه نشان داد كه محل فعاليث افراد و مسافت دورى

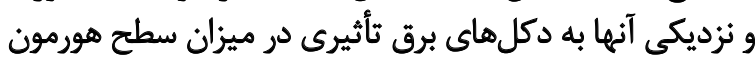

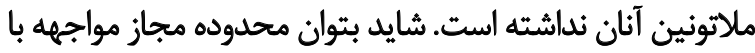

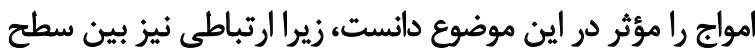

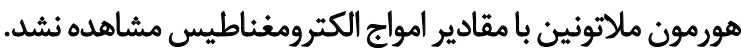

$$
\text { تنيجلمَيرى }
$$

يكى از جنبههاي مهمم مطالعه حاضر را مىتوان يُاسخ دادن به 


\section{Reference}

[1] Brzezinski A. Melatonin in humans. N Engl J Med. 1997; 336(3):186-95. [DOI:10.1056/NEJM199701163360306] [PMID]

[2] Sokolovic D, Djordjevic B, Kocic G, Stoimenov TJ, Stanojkovic $Z$, Sokolovic DM, et al. The effects of melatonin on oxidative stress parameters and DNA fragmentation in testicular tissue of rats exposed to microwave radiation. Adv Clin Exp Med. 2015; 24(3):429-36. [DOI:10.17219/acem/43888] [PMID]

[3] Michaelson SM. Health implications of exposure to radiofrequency/microwave energies. Br J Ind Med. 1982; 39(2):105-19. [DOI:10.1136/oem.39.2.105] [PMID] [PMCID]

[4] Ohayon MM, Stolc V, Freund FT, Milesi C, Sullivan SS. The potential for impact of man-made super low and extremely low frequency electromagnetic fields on sleep. Sleep Med Rev. 2019; 47:28-38. [DOI:10.1016/j.smrv.2019.06.001] [PMID]

[5] Ahlbom A. Neurodegenerative diseases,suicide and depressive symptoms in relation to EMF. Bioelectromagnetics. 2001; (S5):S132-43. [DOI:10.1002/1521-186X(2001)22:5+3.0.CO;2-V]

[6] Carpenter DO. Human disease resulting from exposure to electromagnetic fields. Rev Environ Health. 2013; 28(4):159-72. [DOI:10.1515/reveh-2013-0016]

[7] Sahraei H. [Induction of anger in rhesus monkeys using ELF waves at a frequency of $30 \mathrm{~Hz}$ (Persian)]. Paramed Sci Mil Health. 2019; 14(3):5-5._https://jps.ajaums.ac.ir/article-1-200-fa.html

[8] Juutilainen J, Kumlin T. Occupational magnetic fieldexposure and melatonin: interaction with light-at-night. Bioelectromagnetics. 2006; 27(5):423-6. [DOI:10.1002/bem.20231] [PMID]

[9] El-Helaly M, Abu-Hashem E. Oxidative stress, melatoninlevel, and sleep insufficiency among electronic equipmentrepairers. Indian J Occup Environ Med. 2010; 14(3):66-70. [DOI:10.4103/0019-5278.75692] [PMID] [PMCID]

[10] Yousefi HA, Nasiri P. Psychological effect of occupational exposure to electromagnetic fields. J Res Health Sci. 2006; 6(1):18-21.__http://journals.umsha.ac.ir/index.php/JRHS/article/view/303

[11] Bagheri Hosseinabadi M, Khanjani N, Ebrahimi MH, Haji B, Abdolahfard $M$. The effect of chronic exposure to extremely low-frequency electromagnetic fields on sleep quality, stress, depression and anxiety. Electromagn Biol Med. 2019; 38(1):96101. [DOI:10.1080/15368378.2018.1545665] [PMID]

[12] Daryabari H, Bahramifar A, Morshedi M, Lotfi B. [Investigation of the relationship between exposures to electromagnetic waves with some clinical disorders in radar device users (Persian)]. J Mar Med. 2019; 1(1):18-23. [DOI:10.30491/1.1.18]

[13] Bowman JD, Kelsh MA, Kaune WT. Manual for measuring occupational electric and magnetic fields exposures. U.S. Department of Health and Human Services, Public Health Service, Centers for Disease Control and Prevention, National Institute for Occupational Safety and Health, Division of Biomedical and Behavioral Sciences; 1998.

[14] Ministry of Health and Medical Education.Electronic forms [Internet]. 2017 [Updated 2017]. Available from: http://thc. qums.ac.ir/Portal/home/?177041/\%D9\%81\%D8\%B1\%D9\%85\%D9\%87\%D8\%A7\%DB\%8C-\%D8\%A7\%D9\%84\%DA\%A9\%D8\%A A\%D8\%B1\%D9\%88\%D9\%86\%DB\%8C\%DA\%A9\%DB\%8C
[15] ACGIH. TLVs and BEls based on the documentation of the threshold limit values for chemical substances and physical agents and biological exposure indices. Cincinnati: ACGIH Publication; 2007. https://www.amazon.com/TLVs-BEls-2007-Documentation-Substances/dp/1882417690

[16] International Commission on Non-lonizing Radiation Protection. ICNIRP statement on the guidelines for limiting exposure to time-varying electric, magnetic and electromagnetic fields (up to $300 \mathrm{GHz}$ ). Health Phys. 2009; 97(3):257-8. [DOI:10.1097/HP.0b013e3181aff9db] [PMID]

[17] Mohammadyan M, Alizadeh Larimi A, Gorgani M, Taghavi Soghondikolaei F. [Measurment of electromagnetic field of minitors and electric posts in one of the oil product distribution company in mazandaran province (Persian)]. J Res Environ Health. 2017; 3(2):150-7. [DOI: 10.22038/JREH.2017.25132.1166]

[18] Hosseini SZ, Jalili Jahromi A, Malakootian MA. [Investigation and measurement of electric and magnetic fields In the vicinity of Tehran's metropolitan high-power lines and substations (Persian)]. Twenty-seventh International Conference on Electricity, 2012, Tehran, Iran. https:// civilica.com/doc/178257/

[19] Eskandari M.H., et al. [Examination of 380 and 800 microtesla electromagnetic fields effect on plasma cortisol hormone level and humural immunity in Balb/c mice (Persian)]. Biol J. 2009; 4(2):9-18. https://www. sid.ir/en/journal/ViewPaper.aspx?ID=193443

[20] Ghorbani F, Eshaghi M, Dehghanpor T, Karami Z. [Assessment of Extremely Low Frequency (ELF) electric and magnetic felds in Hamedan high electrical power stations and their effects on workers (Persian)]. Iran J Med Phys. 2011; 8(3):61-71. https://www.sid.ir/fa/journal/ViewPaper.aspx?ID=149146

[21] Mazurek PA, Naumchuk OM, Kot K, Wdowiak A, Zybała M. Exposure of high frequency electromagnetic fields in the living environment. EJMT. 2018; 4(21):33-9. http://www.medical-technologies.eu/upload/exposure_of_high_frequency_electromagnetic_fields_-_mazurek.pdf

[22] Brainard GC, Kavet R, Kheifets LI. The relationship between electromagnetic field and light exposures to melatonin and breast cancer risk: A review of the relevant literature. J Pineal Res. 1999; 26(2):65-100. [DOI:10.1111/j.1600-079X.1999.tb00568.x] [PMID]

[23] Dyche J, Anch AM, Fogler KA, Barnett DW, Thomas C. Effects of power frequency electromagnetic fields on melatonin and sleep in the rat. Emerg Health Threats J. 2012; 5(1):10904. [DOI:10.3402/ehtj. v5i0.10904] [PMID] [PMCID]

[24] Manikonda PK, JagotaA A. Melatonin administration differentially affects age-induced alterations in daily rhythms of lipid peroxidation and antioxidant enzymes in male rat liver. Biogerontology. 2012 13(5):511-24. [DOI:10.1007/s10522-012-9396-1] [PMID]

[25] Farhud D ,Tahavorgar A. [Melatonin Hormone, Metabolism and its clinical effects: A review (Persian)]. Iran J Endocrinol Metab. 2013; 15(2):211-23. https://www.sid.ir/en/journal/ViewPaper. aspx?|D=339250 